\title{
A Prospective Multicenter Registry of Patients Undergoing Hysteroscopic Morcellation of Uterine Polyps and Myomas
}

\author{
Michael D. Scheiber, MD, MPH, and Serena H. Chen, MD²
}

\begin{abstract}
Background: Hysteroscopic morcellation removes uterine pathology under direct visualization with continuous real-time tissue fragment removal. Objective: The aim of this study was to explore the feasibility of hysteroscopic morcellation across a diverse set of facilities, including both surgical and office-based settings. Design: This was a prospective, single-arm, multicenter registry development (Canadian Task Force classification II-3). Materials and Methods: Thirty-four U.S. obstetrics and gynecology facilities enrolled subjects into the registry. Inclusion criteria were women ages 18-65 with indications for hysteroscopic myomectomy and/or polypectomy who were treated with the MyoSure ${ }^{\circledR}$ Hysteroscopic Tissue Removal System (Hologic Inc., Marlborough, MA). Intrauterine lesion type/size and removal parameters, adverse events (AEs), and physician satisfaction ratings were recorded. Results: A total of 559 pathologies (187 fibroids; 372 polyps) were removed from 278 registered subjects (mean age: $43.9 \pm 9.0$ years), with 250 procedures $(89.9 \%)$ performed in an ambulatory surgery center or hospital outpatient setting and $28(10.1 \%)$ in a gynecologic office setting. Most patients $(n=206,74.1 \%)$ were treated for abnormal uterine bleeding, and $42(15.1 \%)$ were treated for infertility. Mean fibroid diameter was $2.2 \pm 1.2 \mathrm{~cm}$. Mean polyp diameter was $1.3 \pm 1.0 \mathrm{~cm}$. Overall mean percentage of pathology removed was $95.4 \%$ (polyps $99.3 \%$, fibroids $86.8 \%$ ). Five AEs included four incidents of blunt cervical trauma and a single postoperative case of pedal edema; all were considered mild and resolved spontaneously. Postprocedure surveys indicated that $95 \%$ of reporting physicians were "satisfied" or "highly satisfied" with device performance. Conclusions: Hysteroscopic morcellation of intrauterine pathology was accomplished safely with a high degree of physician satisfaction in 278 patients treated in diverse healthcare settings that are reflective of general community practice in the United States. (J GYNECOL SURG 32:318)
\end{abstract}

Keywords: intrauterine morcellation, hysteroscopic morcellator, endometrial polyp, submucous myoma

\section{Introduction}

A PPROXIMATELY 30\%-50\% OF ABNORMAL uterine bleeding (AUB) is caused by intracavitary polyps and submucous myomas. ${ }^{1,2}$ There is compelling evidence that removing these growths provides excellent resolution of symptoms. ${ }^{1-3}$ In addition to dilation and curettage and polyp forceps, operative hysteroscopy is among the most common minimally invasive surgical treatments for intrauterine polyp and myoma removal. In electrothermal hysteroscopy, a resectoscope equipped with a radiofrequency-activated cutting loop or an electrical bipolar loop is used to remove intrauterine pathology. ${ }^{4-6}$ Tissue fragments resected from either the polyp or fibroid remain in the distended uterus until manually removed with graspers. As the surgery proceeds, these free-floating tissue pieces can obscure the surgeon's view of the operating site, thereby hindering the procedure. In addition, electrothermal resectoscopy carries a risk of causing inadvertent thermal damage to healthy endometrium that surrounds lesions. ${ }^{7}$ Newer technologies are designed to avert these limitations.

Intrauterine morcellation is a newer approach to operative hysteroscopy that removes uterine pathology under direct visualization with continuous real-time tissue fragment removal. Morcellation instruments use a rotating blade to cut tissue and simultaneously remove resected fragments with uninterrupted irrigation and vacuum aspiration. This approach does not introduce an electrical current inside the uterus and has no risk of potential thermal damage to healthy endometrium.

\footnotetext{
${ }_{2}^{1}$ Institute for Reproductive Health, Cincinnati, OH.

${ }^{2}$ Institute for Reproductive Medicine and Science at Saint Barnabas Medical Center, Livingston, NJ.

(C) Michael D. Scheiber et al., 2016; Published by Mary Ann Liebert, Inc. This Open Access article is distributed under the terms of the Creative Commons License (http://creativecommons.org/licenses/by/4.0), which permits unrestricted use, distribution, and reproduction in any medium, provided the original work is properly credited.
} 
Compared to resectoscopy, hysteroscopic morcellation reduces operative time for removing uterine lesions. Hamerlynck et al. ${ }^{8}$ reported mean operating times for hysteroscopic morcellation of 34 myomas and 278 polyps at 18.2 and 6.6 minutes, respectively, without complications. Rubino and Lukes ${ }^{9}$ reported a mean resection time of $<3$ minutes ( 42 myomas and 76 polyps combined) in 74 patients who underwent morcellation for AUB. Mean pathology removal was nearly complete for both polyps (99.9\%) and myomas (95.9\%), and patients reported significantly reduced symptoms and improved quality of life (QoL) at postoperative 12 months. In a randomized controlled trial (RCT) of residents-in-training, morcellation reduced total operating time for polyp and myoma removal by $72 \%$ and $61 \%$, respectively, versus conventional resectoscopy. ${ }^{10}$ Thus, hysteroscopic morcellation removes intrauterine lesions faster than conventional resectoscopy techniques.

Hysteroscopic myomectomy with an electrothermal loop is conventionally performed in the operating room with the patient under general anesthesia. Researchers have begun assessing the performance of hysteroscopic morcellation in an office versus an ambulatory setting. For instance, Lukes et $\mathrm{al}^{11}$ reported results of 5 institutions that successfully removed a total of 42 polyps and 16 myomas in patients treated in an ambulatory surgical center (ASC, $n=20$ patients) or an office-based setting ( $n=20$ patients). Rubino and Lukes $^{9}$ reported similar outcomes for 32 patients randomized for treatment in an ASC versus 42 patients treated in an office-based setting, with no significant differences in pain scores, adverse events (AEs), patient satisfaction, percentage of pathology removed, or 12-month QoL improvement between ASC and office sites.

Although studies have established the feasibility of hysteroscopic morcellation for polyp and myoma removal in general, most have been restricted to reporting experiences from either a single site or a small number of research-focused institutions. To investigate further the safety and efficacy of hysteroscopic morcellation of intrauterine polyps and myomas in varied community healthcare settings, prospective multicenter studies with larger patient populations are required. To address this need, the current authors established a prospective multicenter registry to assess outcomes better of hysteroscopic morcellation procedures performed in both ASC/hospital outpatient department (HOPD) and office-based settings.

\section{Materials and Methods}

Study subjects had been prospectively registered and enrolled in a multicenter registry. The registry enrolled women ages 18-65 with indications for hysteroscopic myomectomy and/or polypectomy. Inclusion criteria for this study were identification of intrauterine pathology via ultrasound, salineinfusion sonography, or hysteroscopic examination, with polyps of any size and/or submucosal myomas $\leq 6 \mathrm{~cm}$ in diameter. Exclusion criteria were pregnancy, IUD in situ at the time of the procedure, current use of anticoagulant or antiplatelet medication in addition to low-dose aspirin, active pelvic inflammatory disease or pelvic/vaginal infection, known or suspected coagulopathy or bleeding disorder, increased fluidoverload risk (e.g., history of predisposing cardiac, hepatic, or renal dysfunction), or other comorbid condition(s) that, in the opinion of the investigator, could limit the ability to participate or affect the scientific integrity of the study.
Written informed patient consent was obtained from each subject prior to study enrollment. To minimize selection bias, all subjects were enrolled who met the inclusion criteria and consented to take part in the study. All procedures were performed with institutional review board approval at each facility. The study protocol adhered to the tenets of the Declaration of Helsinki and was Health Insurance Portability and Accountability-compliant. Procedures were performed in either an ASC/HOPD, or an office setting.

All morcellation procedures were performed using the MyoSure ${ }^{\circledR}$ Hysteroscopic Tissue Removal System (Hologic Inc., Marlborough, MA). ${ }^{12}$ This device has a side-facing cutter window housing a 2.5 -mm-diameter cutting blade, presenting with an outer bevel that rotates at $8075 \mathrm{rpm}$ and oscillates at 3 cycles/second, within a 3-mm outer tube. The system includes a tissue trap that is connected to the tissue removal device through a regulated vacuum (Fig. 1). Constant regulated isotonic electrolyte solution irrigation maintains target intrauterine distension pressure, with continuous aspiration of resected tissues that are suitable for histopathologic analysis.

Age, height, weight, body mass index (BMI), and menopausal status were recorded for each patient. In addition, the researchers documented the number, size, and location of all pathologies (fibroids and polyps), and the primary reason for treatment for each patient. The primary efficacy endpoint was the percentage of lesions removed. Ancillary endpoints included total procedure time, total cutting time, fluid deficit, and the necessity for mechanical cervical dilation. A 5-point Likert scale evaluated physician postoperative satisfaction with the morcellation system, with a score of 5 indicating "very satisfied," and a score of 1 indicating "very dissatisfied."

The safety endpoint assessed the incidence of AEs observed prior to patient discharge from the recovery room of the treatment facility or reported by the patient after discharge. Study physicians were requested to report the occurrence of specific potential AEs, which were cramping, nausea, pain, hemorrhage, pulmonary distress, cervical tearing, fibroid recurrence, vaginal bleeding, vomiting, fluid overload, infection, reaction to anesthetic agents, and uterine perforation

Patient characteristics and results of procedures performed in an office setting were compared with those performed in ASCs. Continuous variables were compared using $t$-tests. Categorical data and variables recorded as proportions were compared using Fisher's exact test with $2 \times 2$ contingency tables. Analyses software included Excel (Microsoft Corp., Bellingham, WA) and Prism, version 5.0 (GraphPad Inc., San Diego, CA). $p$-Values $<0.05$ were considered indicative of statistically significant differences between comparator groups. Data were analyzed using a modified intention-to-treat basis, and were used to assess all patients who satisfied study enrollment criteria.

\section{Results}

A total of 41 investigators at 34 different institutions treated a total of 278 patients. The participating institutions were diverse with respect to both facility type (7 obstetrics/gynecology offices, 15 nonacademic hospitals/ASCs, 12 academic hospitals/ ASCs) and U.S. geographic location (5 Northeast, 7 Midwest, 13 South, and 9 West). Of 278 total subjects, 250 were treated in an ASC or HOPD setting, and 28 were treated in a gynecology office setting (Table 1). The average number of patients treated by each physician was 8.1 (range: 1-20). The proportion of 


\section{A}
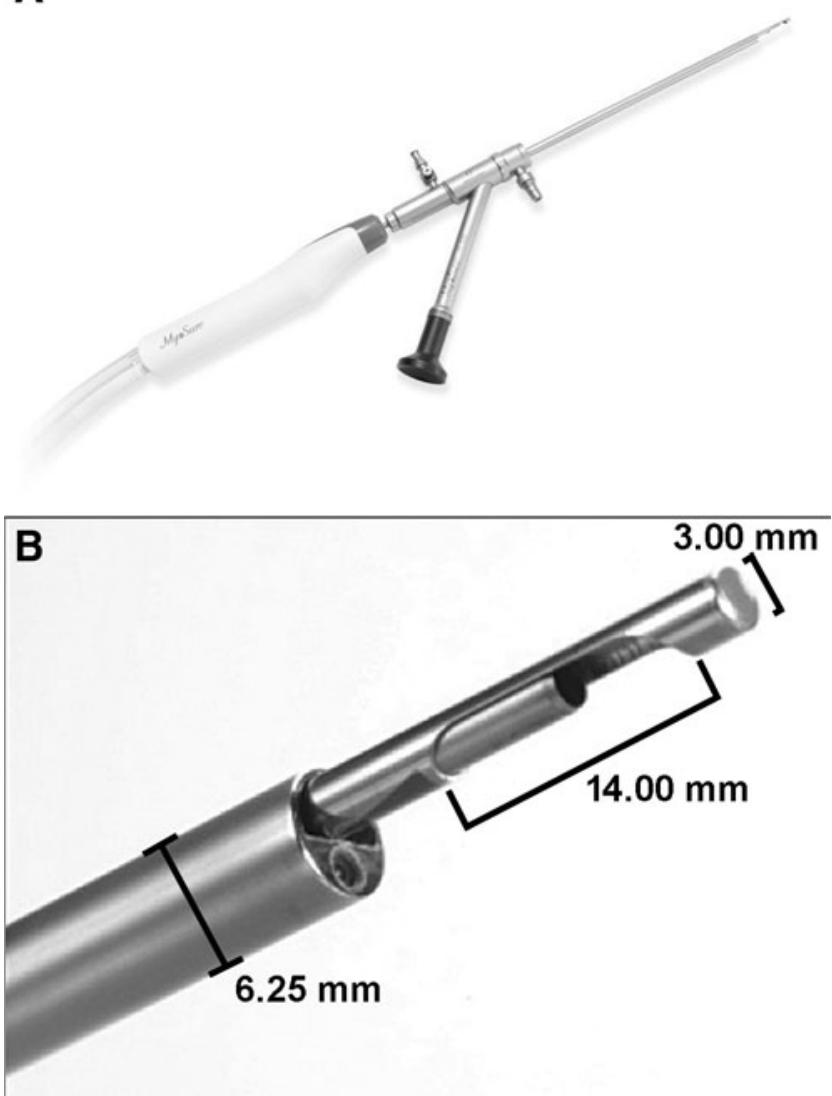

FIG. 1. The MyoSure ${ }^{\circledR}$ Hysteroscopic Tissue Removal System (Hologic Inc., Marlborough, MA) includes (A) the 3.00$\mathrm{mm}$-diameter cutting device that is inserted through the system's small-bore, 6.25-mm-outer diameter (19-French equivalent) hysteroscope housing or other conventional surgical hysteroscope sheaths (typically $7-10 \mathrm{~mm}$, or $21-30$-French equivalent, in outer diameter). (B) Enlarged view of the side-facing cutting window. Under hysteroscopic guidance, the standard unit's cutting blade contacts target tissue through a 14-mm-long and 1.8-mm-deep side channel on the morcellator distal shaft. Foot pedal-controlled activation retracts the blade guard covering the window and engages the stainless steel blade's dual cutting motion, rotating at up to $8075 \mathrm{rpm}$ while oscillating at 3 cycles/ second. The system is available in standard, smaller (LITE), and larger (XL) sizes, with slightly different cutting window dimensions and tissue-removal rates. The standard mid-sized device that was used in $>90 \%$ of procedures is shown.

patients who self-identified as non-Caucasian minority was higher in the ASC/HOPD setting (46\%) than for patients treated in physician offices $(21 \% ; p=0.0156)$. The percentage of patients treated for a known polyp, fibroid, or other intrauterine mass was higher in the office setting (39\%) versus the ASC/ HOPD (14\%; $p=0.0020)$. Most patients $(73.4 \%)$ were premenopausal. Two hundred and six patients $(74.1 \%)$ were treated for AUB, and 42 (15.1\%) were treated for infertility. Additional information regarding patient demographics and physicianreported indications for treatment is provided in Table 1.

The 559 pathologies removed from the current cohort included 187 fibroids (33.5\%) and 372 polyps (66.5\%; Table 2). Mean fibroid diameter was $2.2 \pm 1.2 \mathrm{~cm}$ (range: $0.3-5.5 \mathrm{~cm}$ ). Mean polyp diameter was $1.3 \pm 1.0 \mathrm{~cm}$ (range:
$0.1-7.0 \mathrm{~cm})$. When comparing pathologies from patients treated in the office versus ASC/HOPD setting, there were no significant differences in lesion type or size. Fibroids were most commonly located in the anterior section of the uterus (26.2\% of the total). Polyps were most commonly located in the posterior uterus $(24.2 \%$ of the total).

The overall mean percentage of pathology removed was $95.4 \%$ (Table 3). By lesion type, $99.3 \%$ of polyps and $86.8 \%$ of fibroids were removed. For all subjects, the median reported cutting time was 2.0 minutes (range: 1.0-63.3 minutes), and median fluid deficit was $120 \mathrm{cc}$ (range: 0-2800 cc; Table 3). When surveyed postprocedure, $95 \%$ of responding physicians reported they were either "satisfied" or "highly satisfied" with device performance.

Of 245 subjects for whom cervical dilation information was recorded, mechanical dilation was performed for 215 patients $(87.7 \%)$, and mean dilation was $0.9 \pm 0.5 \mathrm{~cm}$ (range: $0.3-$ $2.8 \mathrm{~cm}$; not shown). General anesthesia was administered in a greater proportion of patients treated in the ASC/HOPD setting (78\%) versus the office setting (7\%; Table 3). Office-based procedures used oral and intravenous sedation and cervical blocks more frequently than ASCs and HOPDs $(p<0.0001$ for each anesthesia type). Mean postoperative recovery time was $54 \%$ longer in the ASC/HOPD setting (57 minutes) than for procedures performed in the office (37 minutes; $p=0.0263$ ).

Five AEs were reported in the 278 subjects; 4 patients had mild cervical trauma and 1 patient experienced a moderate case of postoperative pedal edema. All AEs were considered mild and resolved spontaneously.

\section{Discussion}

The primary endpoint of this study was pathology removal. This study showed a high percentage of removed pathology $(95.4 \%)$ in all patients using hysteroscopic morcellation, with no significant difference between office and ASC/HOPD settings. While postprocedure clinical symptoms were not recorded in this registry, a previous study demonstrated favorable improvement in 1-year clinical symptom and QoL for patients whose fibroids were removed incompletely. ${ }^{9}$ Importantly, residual fibroid tissue left behind after incomplete myoma resection spontaneously regresses in $>50 \%$ of AUB patients with incomplete primary resection, primarily during the first 3 months after intervention, and often in entirety. ${ }^{13}$ Thus, one can expect observable normalization of endometrial morphology and function after uterine lesion morcellation, including in women with limited amounts of residual pathology.

Reported AE rates in this study were low and all AEs were considered mild. This is consistent with previous findings by others, including Emanuel, ${ }^{14}$ who noted that morcellation does not use electrocoagulation and that there is no lateral thermal or electrical energy spread. Instead of thermal coagulation, hemostasis after morcellation occurs by spontaneous myometrial contraction. In addition, because hysteroscopic morcellation removes lesion fragments automatically while inserted into the uterus, the procedure is associated with a decreased number of device transcervical introductions, thereby minimizing the likelihood of cervical trauma and uterine perforations. Researchers who conducted an RCT of residents-in-training reported that morcellation reduced total operating time for polyp and myoma removal by $72 \%$ and $61 \%$, respectively, versus conventional resectoscopy. ${ }^{10}$ Part of this time saving was 
Table 1. Patient Information by Treatment Site Type

\begin{tabular}{|c|c|c|c|c|}
\hline Baseline demographics, mean $\pm S D$ & $\begin{array}{l}\text { All sites } \\
(\mathrm{N}=278)\end{array}$ & $\begin{array}{c}\text { Office } \\
(\mathrm{n}=28)\end{array}$ & $\begin{array}{c}A S C / H O P D \\
(\mathrm{n}=250)\end{array}$ & $p$-Value* \\
\hline Age, years & $43.9 \pm 9.0$ & $45.8 \pm 10.4$ & $43.7 \pm 8.8$ & 0.2412 \\
\hline Height, $\mathrm{cm}$ & $163.8 \pm 11.7$ & $164.3 \pm 6.1$ & $163.6 \pm 12.2$ & 0.7449 \\
\hline Weight, kg & $79.4 \pm 22.1$ & $72.9 \pm 17.6$ & $80.1 \pm 22.4$ & 0.1012 \\
\hline BMI, $\mathrm{kg} / \mathrm{m}^{2}$ & $29.5 \pm 8.2$ & $27.0 \pm 6.4$ & $29.8 \pm 8.4$ & 0.1676 \\
\hline \multicolumn{5}{|l|}{ Race, n $(\%)$} \\
\hline American Indian/Alaska First-Nation & $2(0.7)$ & $0(0)$ & $2(0.8)$ & 0.9999 \\
\hline Asian & $11(4.0)$ & $0(0)$ & $11(4.4)$ & 0.6098 \\
\hline Black/African American & $63(22.7)$ & $2(7)$ & $61(24.4)$ & 0.0537 \\
\hline Hispanic/Latina & $42(15.1)$ & $4(14)$ & $38(15.2)$ & 0.9999 \\
\hline Native Hawaiian/Pacific Islander & $2(0.7)$ & $0(0)$ & $2(0.8)$ & 0.9999 \\
\hline White & $158(56.8)$ & $22(79)$ & $136(54.4)$ & 0.0156 \\
\hline Menopausal status, n (\%) & All sites & Office & $A S C / H O P D$ & \\
\hline Premenopausal & $204(73.4)$ & $18(29)$ & $186(74.4)$ & 0.2636 \\
\hline Perimenopausal & $25(9.0)$ & $2(7)$ & $23(9.2)$ & 0.9999 \\
\hline Postmenopausal & $49(17.6)$ & $8(29)$ & $41(16.4)$ & 0.1092 \\
\hline Reasons for treatment ${ }^{\dagger}, \mathrm{n}(\%)$ & All sites & Office & $A S C / H O P D$ & \\
\hline AUB & $206(74.1)$ & $19(68)$ & $187(74.8)$ & 0.4949 \\
\hline Infertility & $42(15.1)$ & $2(7)$ & $40(16.0)$ & 0.2755 \\
\hline Known polyp/fibroid/uterine mass & $46(16.5)$ & $11(39)$ & 35 (14.0) & 0.0020 \\
\hline Postmenopausal bleeding & $16(5.7)$ & $2(7)$ & $14(5.6)$ & 0.6684 \\
\hline Other ${ }^{*}$ & $9(3.2)$ & $4(14)$ & $5(2.0)$ & 0.0074 \\
\hline
\end{tabular}

*Office versus ASC/HOPD.

†Physicians might have reported more than one reason for treatment per patient.

Other: dysmenorrhea; anemia; enlarged endometrial stripe; breast-cancer history; recurrent pregnancy loss; and cavity-filling defect.

SD, standard deviation; ASC/HOPD, ambulatory surgery center/hospital outpatient department; BMI, body mass index; AUB, abnormal uterine bleeding.

Bold indicates $p$-values $<0.05$.

Table 2. Intrauterine Lesion Parameters, by Treatment Site Type

\begin{tabular}{|c|c|c|c|}
\hline Parameter & $\begin{array}{c}\text { All sites } \\
(\mathrm{N}=278 \text { patients })\end{array}$ & $\begin{array}{c}\text { Office } \\
(\mathrm{n}=28)\end{array}$ & $\begin{array}{c}A S C / H O P D \\
(\mathrm{n}=250)\end{array}$ \\
\hline \multicolumn{4}{|l|}{ Lesion traits ${ }^{*}$, mean $\pm S D$} \\
\hline $\begin{array}{l}\text { Lesions/subject, n } \\
\text { Fibroids/subject, n } \\
\text { Polyps/subject, } \mathrm{n} \\
\text { Fibroid diameter, cm } \\
\text { Polyp diameter, cm }\end{array}$ & $\begin{array}{l}2.0 \pm 1.1 \\
0.7 \pm 1.0 \\
1.3 \pm 1.2 \\
2.2 \pm 1.2 \\
1.3 \pm 1.0\end{array}$ & $\begin{array}{l}2.0 \pm 1.2 \\
0.8 \pm 1.1 \\
1.2 \pm 1.3 \\
1.9 \pm 1.2 \\
1.4 \pm 0.8\end{array}$ & $\begin{array}{l}2.0 \pm 1.1 \\
0.7 \pm 1.0 \\
1.4 \pm 1.2 \\
2.2 \pm 1.2 \\
1.3 \pm 1.0\end{array}$ \\
\hline Fibroid location, $n(\%)$ & All sites & Office & $A S C / H O P D$ \\
\hline $\begin{array}{l}\text { Anterior } \\
\text { Posterior } \\
\text { Fundal } \\
\text { Lower uterine segment } \\
\text { Lateral left } \\
\text { Lateral right } \\
\text { Total }\end{array}$ & $\begin{array}{c}49(26.2) \\
40(21.4) \\
36(19.3) \\
15(8.0) \\
18(9.6) \\
29(15.5) \\
187(100 \%)\end{array}$ & $\begin{aligned} 6 & (27) \\
4 & (18) \\
3 & (14) \\
2 & (9) \\
2 & (9) \\
5 & (23) \\
22 & (100 \%)\end{aligned}$ & $\begin{array}{c}43(26.1) \\
36(21.8) \\
33(20.0) \\
13(7.9) \\
16(9.7) \\
24(14.5) \\
165(100 \%)\end{array}$ \\
\hline Polyp location, $n(\%)$ & All sites & Office & $A S C / H O P D$ \\
\hline $\begin{array}{l}\text { Anterior } \\
\text { Posterior } \\
\text { Fundal } \\
\text { Lower uterine segment } \\
\text { Lateral left } \\
\text { Lateral right } \\
\text { Total }\end{array}$ & $\begin{array}{c}63(16.9) \\
90(24.2) \\
65(17.5) \\
42(11.3) \\
48(12.9) \\
64(17.2) \\
372(100 \%)\end{array}$ & $\begin{aligned} 1 & (3) \\
15 & (45) \\
2 & (6) \\
3 & (9) \\
4 & (12) \\
8 & (24) \\
33 & (100 \%)\end{aligned}$ & $\begin{array}{c}62(18.3) \\
75(22.1) \\
63(18.6) \\
39(11.5) \\
44(13.0) \\
56(16.5) \\
339(100 \%)\end{array}$ \\
\hline
\end{tabular}

*No lesion trait was statistically different between office versus ASC/HOPD settings ( $p>0.05)$. Statistics were not performed to compare frequency of lesion intrauterine locations between groups.

ASC/HOPD, ambulatory surgical center/hospital outpatient department; SD, standard deviation. 
Table 3. Operative Parameters and Observations

\begin{tabular}{lcccccrr}
\hline Parameter* $^{*}$ & All sites & $\mathrm{n}$ & Office & $\mathrm{n}$ & ASC/HOPD & $\mathrm{n}$ & $p$-Value \\
\hline \% Pathology removed, by patient & $95.4 \pm 13.2 \%$ & 278 & $96.8 \pm 14.1 \%$ & 28 & $95.2 \pm 13.1 \%$ & 250 & 0.5436 \\
\% Polyp removed, by lesion & $99.3 \pm 5.8 \%$ & 331 & $99.3 \pm 6.1 \%$ & 27 & $99.9 \pm 0.4 \%$ & 304 & 0.0900 \\
\% Fibroid removed, by lesion & $86.8 \pm 24.1 \%$ & 157 & $94.8 \pm 17.6 \%$ & 18 & $85.8 \pm 24.7 \%$ & 139 & 0.1368 \\
Adverse events, \% of patients & $1.8 \%$ & 278 & $3.6 \%$ & 28 & $1.6 \%$ & 250 & 0.4143 \\
Fluid deficit, cc & $287 \pm 453$ & 234 & $238 \pm 666$ & 17 & $291 \pm 446$ & 217 & 0.6510 \\
Resection time, min & $6.0 \pm 9.0$ & 229 & $8.9 \pm 15.6$ & 21 & $5.8 \pm 8.1$ & 208 & 0.0978 \\
Time in PACU, min & $55.4 \pm 37.1$ & 207 & $36.8 \pm 24.7$ & 16 & $57.0 \pm 37.5$ & 191 & $\mathbf{0 . 0 2 6 3}$ \\
Physician satisfaction score 4-5, \% & $95 \%$ & 278 & $89 \%$ & 28 & $96 \%$ & 250 & 0.1470 \\
Anesthesia type, $n$ (\%) & $6(2.2 \%)$ & $5(18 \%)$ & & $1(<1 \%)$ & $<\mathbf{0 . 0 0 0 1}$ \\
Oral sedation & $41(14.7 \%)$ & $15(54 \%)$ & $26(10.4 \%)$ & $<\mathbf{0 . 0 0 0 1}$ \\
Cervical block & $68(24.5 \%)$ & $17(61 \%)$ & $51(20.4 \%)$ & $<\mathbf{0 . 0 0 0 1}$ \\
IV sedation & $196(70.5 \%)$ & $2(7 \%)$ & $194(77.6 \%)$ & $<\mathbf{0 . 0 0 0 1}$ \\
General anesthesia & & & & & \\
\hline
\end{tabular}

*Values provided as mean \pm standard deviation, or $n(\%)$, as appropriate.

${ }^{\dagger} p$-Values compare office to ASC/HOPD.

ASC/HOPD, ambulatory surgical center/hospital outpatient department; min, minutes; PACU, post-anesthesia care unit; IV, intravenous. Bold indicates $p$-values $<0.05$.

attributed to the reduced number of device insertions during procedures, ranging from 1-2 insertions with morcellation versus 3-50 with conventional resectoscopy. These factors combined to result in fewer complications with hysteroscopic morcellation, compared to electrosurgical resection. Likewise Smith et al. ${ }^{15}$ found that morcellation was significantly quicker than electrosurgical resection for hysteroscopic polypectomy, and was less painful, more acceptable to women, and more likely to remove endometrial polyps completely.

Nearly $50 \%$ of hysteroscopic complications are related to difficulty in traversing the cervix. ${ }^{16}$ The MyoSure operative resectoscope has an outer diameter of $6.25 \mathrm{~mm}$ (19-French), versus the 7-10-mm diameter (21-30-French) of most surgical sheaths. Small-bore hysteroscopes require less cervical dilation for uterine access, and are associated with decreased patient pain and discomfort, fewer procedural complications, and greater patient satisfaction, for both diagnostic ${ }^{17}$ and surgical ${ }^{18}$ applications.

Operative hysteroscopy is still most commonly performed in surgical settings. The current study had similar favorable clinical outcomes regardless of site of service, thus demonstrating the feasibility of performing hysteroscopic morcellation of uterine pathologies in an office-based setting. This finding is supported by earlier studies that demonstrated favorable results for inoffice hysteroscopic morcellation ${ }^{9}$ or other in-office hysteroscopic procedures. ${ }^{19}$ The utility of office-based hysteroscopic morcellation is also supported by a study that demonstrated that low pain scores are achievable using local anesthetic delivered by paracervical-block protocols. ${ }^{10}$ Finally, morcellation devices are compatible with newer fluid-management systems that are more reliable and precise for regulating fluid delivery and retrieval rates, thereby improving patient safety. ${ }^{19-21}$

Subjects in the current study were most commonly treated for AUB or infertility, demonstrating that hysteroscopic morcellation can be used for diverse clinical indications. This registry included $>250$ women treated at geographically diverse academic and community-based facilities. With $>30$ office and surgical sites, and 40 investigators of varying levels of experience contributing results, this study provides a current and representative depiction of hysteroscopic morcellation used in routine practice across the United States. This study supports the feasibility of using hysteroscopic morcellation to treat intrauterine pathology in a wide range of clinical settings.

The strong points of this study are its prospective nature, the relatively large overall cohort size, and the diversity in geographic locales and nature of participating healthcare facilities. The primary study limitation was that the single-arm design did not compare outcomes between hysteroscopic morcellation and other uterine pathology treatments, such as resectoscopy. Another limitation was the relatively small size of the officebased cohort arm. Expansion of the currently established registry will complement new and ongoing RCTs in assessing the utility of hysteroscopic morcellation for ameliorating intrauterine pathologies safely in both office and ASC settings.

\section{Conclusions}

This study demonstrated the successful use of hysteroscopic morcellation of uterine fibroids and polyps in $>250$ patients treated in settings reflective of general community practice in the United States. Hysteroscopic morcellation is a feasible approach for removing intrauterine lesions in both ASC/HOPD and office-based settings, has an excellent safety profile, and provides a high level of physician satisfaction.

\section{Acknowledgments}

The authors thank Matt Silverman, $\mathrm{PhD}$ (Writing Assistance Inc., Plymouth, MN), and Lloyd Zimmerman, MD, MPH (Zimmerman Medical Communications, New York, NY), for their assistance in preparing the manuscript for this article.

\section{Author Disclosure Statement}

M.D.S. and S.H.C. received compensation for speaking engagements by Hologic Inc. Neither author has any other potential conflicts of interest to report, financial or otherwise.

\section{References}

1. Stewart EA. Uterine fibroids. Lancet 2001;357:293.

2. Lumsden MA and Abukhnjr S. Clinical presentation of uterine fibroids. In: Radiological Interventions in Obstetrics 
and Gynaecology. Reidy J, Hacking N, McLucas B, eds. Berlin: Springer, 2014:29.

3. American Association of Gynecologic Laparoscopists (AAGL). AAGL Practice report: Practice guidelines for the diagnosis and management of endometrial polyps. J Minim Invasive Gynecol 2012;19:3.

4. Sesti F, Marziali N, Santomarco N. Hysteroscopic surgery for endometrial polyps using a bipolar microelectrode. Int J Gynaecol Obstet 2000;71:283.

5. Golan A, Sagiv R, Berar M, Ginath S, Glezerman M. Bipolar electrical energy in physiologic solution-a revolution in operative hysteroscopy. J Am Assoc Gynecol Laparosc 2001;8:252.

6. Vilos GA. Intrauterine surgery using a new coaxial bipolar electrode in normal saline solution (Versapoint): A pilot study. Fertil Steril 1999;72:740.

7. Touboul C, Fernandez H, Deffieux X, Berry R, Frydman R, Gervaise A. Uterine synechiae after bipolar hysteroscopic resection of submucosal myomas in patients with infertility. Fertil Steril 2009;92:1690.

8. Hamerlynck TW, Dietz V, Schoot BC. Clinical implementation of the hysteroscopic morcellator for removal of intrauterine myomas and polyps: A retrospective descriptive study. Gynecol Surg 2011;8:193.

9. Rubino RJ, Lukes AS. Twelve-month outcomes for patients undergoing hysteroscopic morcellation of uterine polyps and myomas in an office or ambulatory surgical center. $\mathbf{J}$ Minim Invasive Gynecol 2015;22:285.

10. van Dongen H, Emanuel MH, Wolterbeek R, Trimbos JB, Jansen FW. Hysteroscopic morcellator for removal of intrauterine polyps and myomas: A randomized controlled pilot study among residents in training. J Minim Invasive Gynecol 2008;15:466.

11. Lukes AS, Roy KH, Presthus JB, Diamond MP, Berman JM, Konsker KA. Randomized comparative trial of cervical block protocols for pain management during hysteroscopic removal of polyps and myomas. Intl J Womens Health 2015:7:833.
12. Cohen S, Greenberg JA. Hysteroscopic morcellation for treating intrauterine pathology. Rev Obstet Gynecol 2011;4:73.

13. Dueholm M, Forman A, Ingerslev J. Regression of residual tissue after incomplete resection of submucous myomas. Gynaecol Endosc 1998;7:309.

14. Emanuel MH. New developments in hysteroscopy. Best Pract Res Clin Obstet Gynaecol 2013;27:421.

15. Smith PP, Middleton LJ, Connor M, Clark TJ. Hysteroscopic morcellation compared with electrical resection of endometrial polyps: A randomized controlled trial. Obstet Gynecol 2014;123:745.

16. Al-Fozan H, Firwana B, Al Kadri H, Hassan S, Tulandi T. Preoperative ripening of the cervix before operative hysteroscopy. Cochrane Database Syst Rev 2015;4:CD005998.

17. Pluchino N, Ninni F, Angioni S, et al. Office vaginoscopic hysteroscopy in infertile women: Effects of gynecologist experience, instrument size, and distention medium on patient discomfort. J Minim Invasive Gynecol 2010;17:344.

18. Dealberti D, Riboni F, Prigione S, Pisani C, Rovetta E, Montella F, Garuti G. New mini-resectoscope: Analysis of preliminary quality results in outpatient hysteroscopic polypectomy. Arch Gynecol Obstet 2013;288:349.

19. Wortman M, Daggett A. Operative hysteroscopy in an officebased surgical setting: Review of patient safety and satisfaction in 414 cases. J Minim Invasive Gynecol 2013;20:56.

20. Cooper NA, Clark TJ. Ambulatory hysteroscopy. Obstet Gynaecol 2013;15:159.

21. Lieng M, Istre O, Qvigstad E. Treatment of endometrial polyps: A systematic review. Acta Obstet Gynecol 2010;89:992.

Address correspondence to: Michael D. Scheiber, MD, MPH 3805 Edwards Road, Suite 450 Cincinnati, OH 45209

E-mail: scheibermd@gmail.com 